\title{
Age related retinal Ganglion cell susceptibility in context of autophagy deficiency
}

\author{
Katharina Bell ${ }^{1,2}$, Ines Rosignol ${ }^{1}$, Elena Sierra-Filardi ${ }^{1}$, Natalia Rodriguez-Muela ${ }^{1,3}$, Carsten Schmelter ${ }^{2}$, \\ Francesco Cecconi ${ }^{4}$, Franz Grus $^{2}$ and Patricia Boya $\mathbb{1 0}^{1}$
}

\begin{abstract}
Glaucoma is a common age-related disease leading to progressive retinal ganglion cell (RGC) death, visual field defects and vision loss and is the second leading cause of blindness in the elderly worldwide. Mitochondrial dysfunction and impaired autophagy have been linked to glaucoma and induction of autophagy shows neuroprotective effects in glaucoma animal models. We have shown that autophagy decreases with aging in the retina and that autophagy can be neuroprotective for RGCs, but it is currently unknown how aging and autophagy deficiency impact RGCs susceptibility and survival. Using the optic nerve crush model in young and olWelcome@1234d Ambra $1^{+/ g t}$ (autophagy/beclin-1 regulator $1^{+/ 9 t}$ ) mice we analysed the contribution of autophagy deficiency on retinal ganglion cell survival in an age dependent context. Interestingly, old Ambra ${ }^{+/ g t}$ mice showed decreased RGC survival after optic nerve crush in comparison to old $\mathrm{Ambral}^{+/+}$, an effect that was not observed in the young animals. Proteomics and mRNA expression data point towards altered oxidative stress response and mitochondrial alterations in old Ambra ${ }^{+/ g t}$ animals. This effect is intensified after RGC axonal damage, resulting in reduced oxidative stress response showing decreased levels of $\mathrm{NqO}$, as well as failure of Nrf2 induction in the old Ambra1 ${ }^{+/ g t}$. Old Ambra $1^{+/ g t}$ also failed to show increase in Bnip3/ and Bnip3 expression after optic nerve crush, a response that is found in the Ambra1 ${ }^{+/+}$ controls. Primary RGCs derived from Ambra $1^{+/ 9 t}$ mice show decreased neurite projection and increased levels of apoptosis in comparison to Ambra1 $1^{+/+}$animals. Our results lead to the conclusion that oxidative stress response pathways are altered in old $A \mathrm{mbra}^{+/ 9 t}$ mice leading to impaired damage responses upon additional external stress factors.
\end{abstract}

\section{Introduction}

Glaucoma are a group of diseases leading to progressive optic nerve damage and accompanying visual field loss, caused by retinal ganglion cell (RGC) death. This multifactorial, neurodegenerative disease is one of the leading causes for blindness and although estimating worldwide prevalence for all glaucoma cases is challenging, we know that glaucoma will affect at least 111.8 million people by the

\footnotetext{
Correspondence: Katharina Bell (kbell@eye-research.org) or

Patricia Boya (patricia.boya@csic.es)

'Department of Cellular and Molecular Biology, Centro de Investigaciones

Biológicas Margarita Salas, CSIC, Madrid, Spain

${ }^{2}$ Experimental and Translational Ophthalmology, Medical Center of the

Johannes Gutenberg University, Mainz, Germany

Full list of author information is available at the end of the article

Edited by R. Killick
}

year $2040^{1}$. The risk for developing glaucoma increases with age and prevalence rapidly rises after the age of 40 years ${ }^{1}$. Next to the clinically most relevant risk factors such as increased intraocular pressure and ageing, one major factor associated with RGC death in glaucoma is mitochondrial dysfunction, which was recently confirmed in a proteomics study analysing human retinal post-mortem glaucoma samples $^{2-4}$.

Autophagy is an important cell catabolic pathway necessary for the recycling of intracellular components via their delivery to the lysosome ${ }^{5}$. During autophagy the material targeted for degradation is packed into a double membrane structure, the autophagosome, which then can fuse with the lysosome for cargo degradation ${ }^{6}$. Not only soluble cell fractions such as proteins are degraded via autophagy, but also

\section{(c) The Author(s) 2020}

(c) (i) Open Access This article is licensed under a Creative Commons Attribution 4.0 International License, which permits use, sharing, adaptation, distribution and reproduction cc) in any medium or format, as long as you give appropriate credit to the original author(s) and the source, provide a link to the Creative Commons license, and indicate if changes were made. The images or other third party material in this article are included in the article's Creative Commons license, unless indicated otherwise in a credit line to the material. If material is not included in the article's Creative Commons license and your intended use is not permitted by statutory regulation or exceeds the permitted use, you will need to obtain permission directly from the copyright holder. To view a copy of this license, visit http://creativecommons.org/licenses/by/4.0/. 
organelles, such as mitochondria, during selective autophagy. In this process cargo receptors and adaptors determine specificity for organelles and other cargo for degradation ${ }^{7}$. Autophagy levels decline with age in many tissues including the brain ${ }^{8-11}$ which contributes to the pathogenesis of various, if not all age-related neurodegenerative diseases ${ }^{12-14}$. Our data in the aging retina also supports this notion and we find decreased mRNA expression of several autophagy regulators such as Atg7 and Beclin1 that results in decreased autophagy flux from 12 months of age onwards in the mouse retina ${ }^{11}$. Other studies have demonstrated the importance of autophagy for proper neuronal development and embryonic survival ${ }^{15,16}$. Programmed cell clearance in early stages of retinal development is dependent on autophagy ${ }^{17}$ and we have shown that mitophagy is important for RGC development, leading to the metabolic shift towards glycolysis which is essential for retinal ganglion cell neurogenesis ${ }^{18}$. Genetic studies show a link between glaucoma and autophagy related genes ${ }^{19}$. Of the genes that have been found associated with glaucoma in genome-wide association studies (GWAS) studies $O p t n^{20}, T b k 1^{21}$, and $O p a-1^{22-24}$ play a role in mitochondrial function and also in mitophagy pathways ${ }^{25-28}$. Interestingly, these genes are supposed to play a role in normal tension glaucoma (NTG) rather than primary open angle glaucoma (POAG), which is, in contrast to NTG, associated with elevated intraocular pressure. Approximately $30-40 \%$ of glaucoma patients suffer from $\mathrm{NTG}^{29}$.

In this study we analysed the effect of ageing and autophagy deficiency on RGC survival using Ambra1 ${ }^{+/ g t}$ mice. AMBRA1 (autophagy/beclin-1 regulator 1) plays a role in the initiation of the autophagy process ${ }^{30}$. It also is a relevant mitophagy receptor that can act in a PINK1/PARKIN dependent and independent way $^{31-33}$. Ambra $1^{\text {gt/gt }}$ mice display embryonic lethality at E10-E14.5 associated with significant neural tube defects $^{30}$. Ambra1 ${ }^{+/ g t}$ animals have been used as a model of subtle autophagy deficiency in many studies including our own, where we show alterations in differentiation of neural stem cells ${ }^{34}$. In this study we find that aged $A m b r a 1^{+/ g t}$ mice have increased susceptibility to axonal insult in vivo in a model of optic nerve crush (ONC). Proteomic analysis shows mitochondrial alterations and changes in the oxidative stress response in the $\mathrm{Ambra1}^{+/ g t}$ animals that could increase the susceptibility to RGC damage.

\section{Materials and methods}

Animals procedures and optic nerve crush (ONC) surgery

5-6 months (young) and 12-14 months (old) $\mathrm{Ambra}^{+/+}$ as well as $A m b r a 1^{\mathrm{gt} /+}$ mice with a CD1 background were used for these experiments. The ratio male/female in all experiments was 1:1 and we could not detect any apparent changes in behaviour of the female mice in our colony. Animals were housed, cared for and euthanized in accordance with European Union guidelines and experiments were approved by the CIB ethics committee for animal experimentation. The mice were housed in a $12 \mathrm{~h} / 12 \mathrm{~h}$ light/dark environment and fed ad libitum. AMBRA1 mutant mice were provided by Francesco Cecconi, University of Rome Tor Vergata ${ }^{30}$. The animals were genotyped by processing tail bud tissue. DNA was isolated from tissue using the NzyTech gDNA Isolation kit (NZYTech) and PCR was performed as previously described ${ }^{30}$. In preparation for the ONC the mice were anaesthetised with a weight adapted intraperitoneal (i.p.) injection of $80 \mathrm{mg} / \mathrm{kg}$ ketamine chlorhydrate solution (Merial, Barcelona, Spain) and $10 \mathrm{mg} / \mathrm{kg} 2 \%$ xylacine chlorhydrate (Bayer, Barcelona, Spain). For surgery, the body temperature of the animals was maintained by placing them on a $37.5^{\circ} \mathrm{C}$ heating platform.

ONC was performed on the left eye. Using $2.5 \mathrm{~mm}$ Vannas Spring Scissors (Fine Science Tools), the conjunctiva and tenon was opened from the 1 o'clock to the 3 o'clock position. By slightly rotating the eyeball nasally and using fine forceps the optic nerve was carefully exposed. Using self-closing negative action tweezers (Dumoxel Dumont Negative Action Tweezer, Style N7) the optic nerve was crushed for $7 \mathrm{~s}$ at a site between 1 and $2 \mathrm{mms}$ behind the globe. During surgery both eyes were moisturised with artificial eye drops, which was continued on the non-surgery eye until the animals woke up. After surgery Ofloxacin eye ointment was applied to the left, surgery eye and all mice received an intraperitoneal injection of Buprenorphine (Buprenorphine; Bedford Laboratories, Bedford, OH, USA). Depending on the experiment, the mice were sacrificed $3,4,7$, or 10 days after the ONC (indicated for each experiment) via cervical dislocation after a brief isoflurane sedation.

\section{Histological evaluation}

The eyes were enucleated after cervical dislocation. For flatmount preparation, the eyes were opened through the cornea, the lens and vitreous body were extracted. Four petal cuts were performed in the retina, which was then mounted on nitrocellulose with the photoreceptors facing down. After fixing in $4 \%$ PFA $1 \mathrm{~h}$ at RT and washing with PBS, half of the flatmount was used for RGC counts and the other half of the retina was embedded in OCT and cryosections were prepared as previously described ${ }^{35}$. For RGC counts, the flatmounts were stained for GammaSynuclein (Abnova, H00006623-M01; 1:100, RGC marker) and Calbindin (Sigma, C2724; amacrine cells, 1:100) over night at $4{ }^{\circ} \mathrm{C}$ in a humid chamber. Brn3a staining in the cryosections was performed as previously published ${ }^{36}$. To stain RGCs with Brn3a in the cryosections, the sections were re-fixed with $4 \%$ PFA for $10 \mathrm{~min}$, washed $3 \times$ with PBS for $10 \mathrm{~min}$, then treated with $10 \mathrm{mM}$ citrate buffer $\mathrm{pH}$ 6.0, heated in the microwave, and subsequently permeabilized with $1 \%$ Triton $4 \times 20$ min. After washing, the sections were blocked with BGT (0.3\% BSA, 0.75\% 
glycine, $0.25 \%$ triton $\mathrm{X}-100$ ) for $1 \mathrm{~h}$ and then the primary antibody (anti-Brn3a, Millipore, MAB1585) was incubated over night at $4{ }^{\circ} \mathrm{C}$. The following day, appropriate secondary antibodies (Molecular Probes, anti-rabbit, and anti-mouse) were incubated for $1 \mathrm{~h}$ at RT. The flatmounts were mounted in Fluoromount-G (Cultek, 100-01) with DAPI 1:1000 (D9542, Sigma).

Pictures of the ganglion cell layer of the flatmounts as well as the cryosections were taken with a confocal microscope (TCS SP5; Leica Microsystems, Barcelona, Spain). Masked cell counting was performed using the Image J software $(\mathrm{NIH}$, Bethesda, MD, USA) on maximal confocal projections. For the flatmounts 4-6 fields per half retina were acquired with a X63 objective. Hereby the cells only stained with GammaSynuclein were accounted as RGCs. For the Brn3a staining in the cryosections 12-16 pictures from eight sections covering all regions of the eye were taken per retina.

\section{Quantitative RT-PCR}

Retinal mRNA levels were measured from 13-monthold $A m b r a 1^{+/ g t}$ and $A m b r a 1^{+/+} 3$ and 7 days after ONC ( $n=4-5$ per group). Total RNA from retinas was extracted using TRIzol reagent (Invitrogen), and reverse transcription performed using the High-Capacity cDNA Reverse Transcription Kit (Applied Biosystems, Waltham, MA, USA) according to the manufacturer's instructions. Quantitative real-time PCR was performed with $10 \mathrm{ng}$ cDNA in a Light Cycler 480 Instrument (Roche, Mannheim, Germany) with LightCycler ${ }^{\circ} 480$ probes master mix (Roche, Mannheim, Germany) using Taqman assays (Life Technologies, Carlsbad, CA, USA). TaqMan Gene expression arrays for Mfn2 (Mm00500120_m1), Optn (Mm01333245_m1), Park2 (Mm00450187_m1), Pink1 (Mm00550827_m1), Gpx1 (Mm00656767_g1), Nqo1 (Mm01253561_m1), Bnip3 (Mm01275600_g1), Bnip3l (Mm00786306_s1), Nfe2l2 (Mm00477784_m1), Вecn1 (Mm01265461_m1). Assays were performed in duplicate, and results were normalised according to expression levels of 18s RNA (Hs99999901_s). Ratios were calculated using the contralateral eye.

\section{Proteomics}

Retinae from 13-month old Ambra1 ${ }^{+/ g t}$ and $\mathrm{Ambra1}^{+/+}$ animals were isolated and stored at $-80^{\circ} \mathrm{C}$ until further use $(n=6)$. Homogenisation and further handling of the tissue was performed as previously described in detail ${ }^{37}$. Briefly, the retinae were homogenised in lysis buffer $(400 \mu \mathrm{l} \mathrm{T}$-PER Tissue protein Extraction Reagent/Retina (Thermo Fischer, 78510) using a Precellys 24 homogeniser with 1.4 and $2.8 \mathrm{~mm}$ ceramic balls. Afterwards, the retinal homogenate was exchanged into $300 \mu \mathrm{l}$ phosphate-buffered saline (PBS) using an Amicon $3 \mathrm{kDa}$ centrifugal filter device (Millipore, Billerica, MA, USA). Protein concentration was determined with a BCA protein assay kit (Thermo Fisher Scientific,
Rockford, IL, USA) according to manufacturer's instructions and measured with a Multiscan Ascent photometer (Thermo Fisher Scientific, Rockford, IL, USA) at a wavelength of $570 \mathrm{~nm}$. For the SDS gel protein separation, 2 retinas were pooled (resulting in $n=3$ for WT and HT) and $50 \mu \mathrm{g}$ of each pooled sample was loaded per lane and separated with 10-well NuPAGE 12\% Bis-Tris minigels (Thermo Fisher Scientific, Rockford, IL, USA) under reducing conditions. In gel tryptic digestion was performed as described in detail previously and SOLA $\mu^{\mathrm{TM}}$ HRP Spin plates were used for peptide purification prior to LC-MS/ MS analysis with a Rheos Allegro Pump downscaling to a capillary HPLC system and coupled to a hybrid linear ion trap-Orbitrap MS system (LTQ Orbitrap XL; Thermo Fischer Scientific, Rockfeld, IL USA). Elution gradient of the HPLC and MS-specific parameters are described elsewhere in detail ${ }^{38}$. Parameters used for elution gradient and MS parameters were applied as described previously ${ }^{39,40}$. Data analysis was performed using Perseus software package version 1.6.5. $0^{41}$. LFQ intensities were $\log 2$ transformed and ANOVA was performed. For protein interaction analysis STRING software version 11.0 was used.

\section{RGC culture}

Postnatal day 1 (P1) Ambra1 ${ }^{+/ g t}$ and Ambra1 $1^{+/+}$ littermates were used to isolate RGCs. The eyes were extracted and the retina isolated. The retina was dissociated using the Papain kit from Worthington (PDS LK003150l). Then 25,000 retinal cells were incubated in 96-well plates (Screenstar microplate, Greiner Bioone, 655866), pre-incubated with poly-L-lysine and laminin, with neurobasal A (ThermoFisher, 21103049) medium containing 2\% B27 (ThermoFisher, 17504044), 0.5\% gentamicin (Gibco, 15710-049), 0.25\% L-glutamine (Gibco, 25030-024). Cells were incubated up to 3 days. Light microscopic pictures were taken after 1 and 3 days in vitro (DIV) and axonal neurites were measured manually in masked pictures. Five images were taken per well $(n=15$ per time point). DAPI (Sigma, D9542; 1:1000) staining was performed to determine the total number of cells as well as the number of apoptotic nuclei, which were assessed for each condition in a masked manner. Neuronal Class III Beta-Tubulin (TUJ1) (Biolegend, MMS-435P at 1:500) staining was performed to analyse the \% of RGCs in the culture.

\section{Statistics}

Prism *Version 8.3.19 was used for statistical analysis. Mann-Whitney tests or Krusak-Wallis ANOVA were performed. $p$-values $<0.05$ were estimated to be statistically significant. Graphs were performed with GraphPad Prism (Version 8.3.1) and represent \pm SD or \pm SEM (indicated in the figure legends). 


\section{Results}

Decreased RGC survival in aged autophagy deficient Ambra $1^{+/ g t}$ mice

The ONC is a model to mimic RGC death through IOP independent optic nerve damage. It therefore relates more to glaucoma without elevated eye pressure than POAG. As NTG has been linked to genes involved in autophagy and mitophagy, and is an age-related disease, we aimed to detect changes in RGC loss after ONC depending on age and autophagy impairment. For this, Ambra1 ${ }^{+/ g t}$ animals and Ambra1 $1^{+/+}$littermate controls were subject to ONC, as AMBRA1 is involved in autophagy induction and serves as a mitophagy receptor. After ONC, the left eye was assessed at different times point after injury. To determine the number of RGCs, both the right and the left retina were flatmounted and stained with Gamma-Synuclein and Calbindin. GammaSynuclein staining serves as robust RGC marker in control and damage conditions ${ }^{42}$, however also stains displaced amacrine cells ${ }^{43}$. Although Gamma-Synuclein staining appears weaker in displaced amacrine cells, and therefore could be distinguished, Calbindin staining was used to identify these cells. Cells only stained for Gamma-Synuclein were counted as RGC. As expected, we found a steady, significant decrease of RGCs after ONC, Fig. 1a. RGC numbers decline after ONC in comparison to the respective control eye, from day 4 until day 10 after ONC, Fig. 1b. Seven days post-ONC was chosen as endpoint for the further experiments. We then compared RGC counts of young and old Ambra1 $^{+/+}$and Ambra1 $1^{+/ g t} 7$ days after ONC. When comparing young $A m b r a 1^{+/+}$and $A m b r a 1^{+/ g t}$, we found a significant decrease in RGCs after ONC in both groups but no difference in amount of RGC loss Fig. 1c. In contrast, RGC counts after ONC in the aged animals showed significantly reduced RGC numbers in the $A m b r a 1^{+/ g t}$ animals than in the Ambra1 $1^{+/+}$mice, Fig. 1d. Retinal cryosections and Brn3a staining also showed significantly decreased RGC numbers in $\mathrm{Ambra1}^{+/ \mathrm{gt}}$ animals after $\mathrm{ONC}$ in comparison to Ambra1 $1^{+/+}$, Fig. 1e. These data show an age-dependent decline of RGC survival in an autophagy deficient model.

\section{Altered mitochondrial and neuronal proteins in $\mathrm{Ambra}^{+/ g t}$ retinae}

As we found differences between RGC survival in old $A m b r a 1^{+/ g t}$ and $A m b r a 1^{+/+}$animals, we wanted to explore the molecular players governing this interesting differential RGC survival between these two animal groups. Applying a bottom-up proteomic approach using a HPLC- LTQ Orbitrap XL MS setup, we aimed to understand relevant proteomic changes between old $A m b r a 1^{+/ g t}$ and $\mathrm{Ambra1}^{+/+}$. We identified 1272 proteins in the retinae of the 13-month-old animals, using a false discovery rate $<1 \%$ as filtering criteria. Seventy-one proteins were significantly differently regulated in $A m b r a 1^{+/ g t}$ in comparison to $A m b r a 1^{+/+}$animals, Fig. 2a. Ingenuity Pathway
Analysis (IPA, Quiagen) found changes in glutathione depletion, cell death as well as mitochondrial dysfunction to be among the highest scores, Fig. 2b. 10 of the 71 proteins were mitochondrial proteins, and also 6 of them involved in mitochondrial organisation, 6 proteins were associated with the oxidative stress response, 13 were associated with building neuronal projections and neuronal proteins, 7 of the proteins are associated with eye development, and we also found 2 proteins involved in mitochondrial axonal transport, Fig. 2c. String analysis of these proteins showed a complex protein interaction network with a PPI-value of $2.16 \mathrm{e}-06$, indicating that these proteins have significantly more interactions among themselves than a random set of the same amount of proteins and that these proteins are biologically connected as a group, Supp Fig. 1. Additionally, we found three crystallins (CRYBB2, CRYAA, and CRYAB) to be significantly downregulated in the retinae of the old Ambra1 ${ }^{+/ g t}$ animals, Fig. 2d. IPA revealed phagosome maturation to be one of the most significantly altered canonical pathways ( $p$-value:10.0E-04), including significantly decreased ATP6V1A expression, Suppl. Fig. 2. These data suggest alterations in the mitochondrial function, oxidative stress response, and downregulation of crystallins, a family of chaperones that has important cytoprotective functions in the eye $\mathrm{e}^{44,45}$ and has been linked to the regulation of lysosomal acidification via ATP6V1A.

\section{Impaired oxidative stress response after ONC in Ambra $1^{+/ g t}$ animals}

As oxidative stress and mitochondrial damage were the main pathways altered, we next decided to follow up in this pathway. Given the reported connections between NFE2L2, mitochondria disfunction and oxidative stress response $\mathrm{e}^{46,47}$,we performed $\mathrm{GPCR}$ analysis studying $\mathrm{Nfe} 2 \mathrm{l2}$ (also known as Nrf2), and its downstream factors Nqo1 (NAD(P)H Quinone Dehydrogenase 1) and Gpx1 (Glutathione peroxidase 1) in the retinae 3 and 7 days after ONC in $\mathrm{Ambra1}^{+/+}$and $\mathrm{Ambra1} 1^{+/ \mathrm{gt}}$ animals compared to the contralateral control eyes. $N f e 2 l 2$ showed a significant increase 7 days after ONC in $A m b r a 1^{+/+}$ animals, whereas the expression levels were not altered in the $A m b r a 1^{+/ g t}$ animals, Fig. 3. Last we detected a significant increase of Gpx1 3 days after $\mathrm{ONC}$ in the Ambra1 $1^{+/ g t}$ animals, however lower levels 7 days after ONC, Fig. 3. All these data support our proteomics data indicating that old Ambra1 $1^{+ \text {gt }}$ mice have an impaired retinal oxidative stress response after RGC damage.

\section{Altered mRNA mitophagy regulators levels in Ambra1 ${ }^{+/ g t}$ mice after ONC}

Extensive evidences in the literature have found increased oxidative stress markers, such as in the serum of patients, associated to glaucoma ${ }^{48}$ and in animal models of this 
A

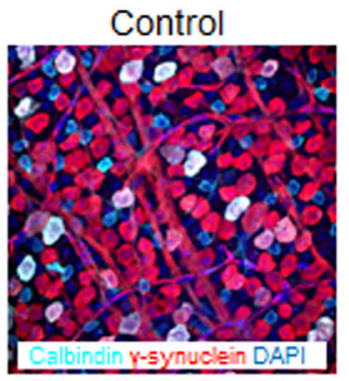

$\mathrm{B}$

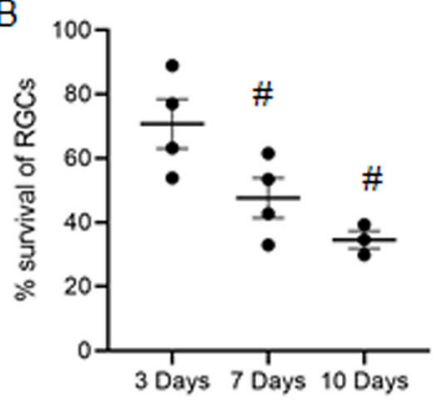

4 days
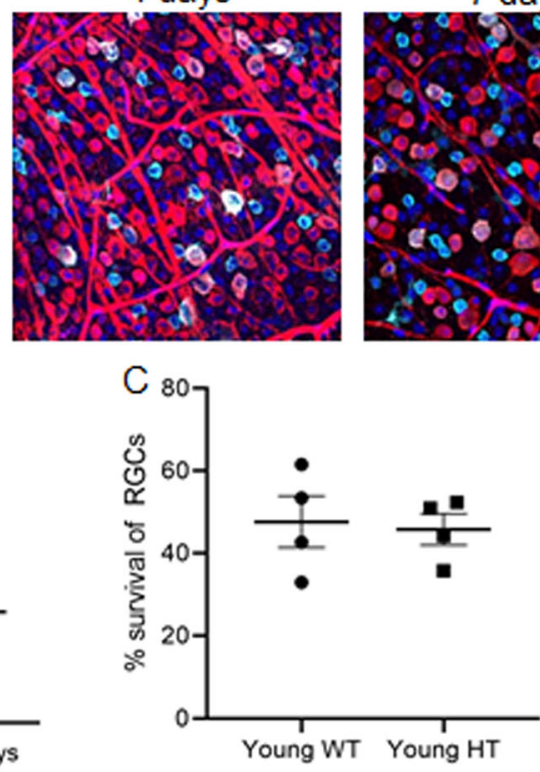

$\mathrm{E}$
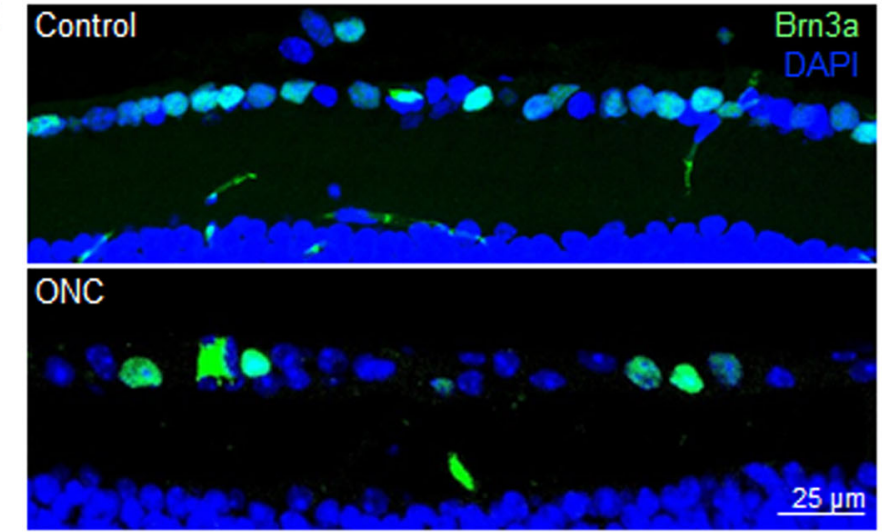

7 days
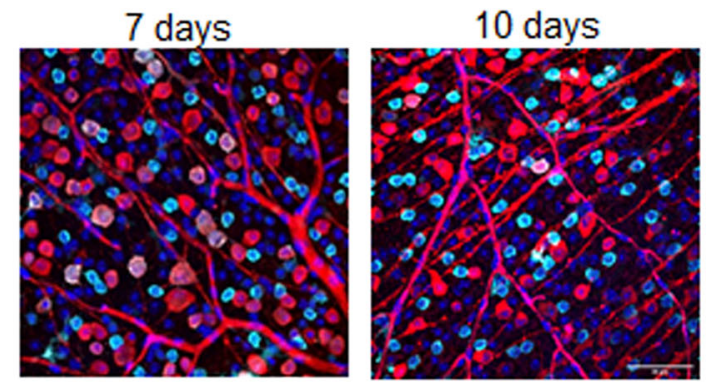

D 80

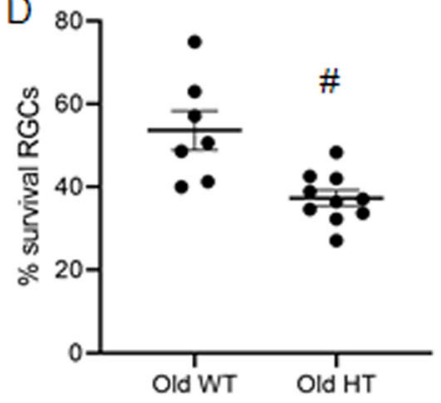

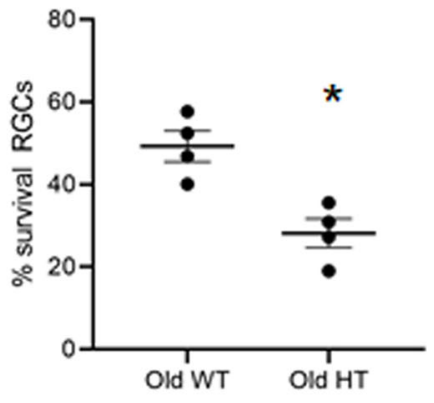

Fig. 1 Old $\mathrm{Ambra}^{+/ \mathrm{gt}}$ mice display reduced RGC survival after ONC. a Immunostaining of retinal ganglion cells in flat mounts at different time points after ONC showing Y-Synuclein (red) and Calbindin (cyan) staining. b \% of RGCs throughout a time course up to 10 days after ONC in young Ambra ${ }^{+/+}$(WT) animals. The number of RGCs was calculated by subtracting from the amount of $\gamma$-Synuclein positive cells minus the displaced amacrine double stained $y$-Synuclein and Calbindin cells. The control eyes are represented as baseline value, $n=4$ animals per group. ${ }^{*} p<0.01$ in comparison to the corresponding non-injured eye. c \% survival of RGCs in 3-months-old (young) Ambra ${ }^{+/+}$and Ambra ${ }^{+/ g t}$ mice 7 days after ONC, $n=4$ animals per group. $\mathbf{d}$ Graph showing the survival of RGCs in old (13-months-old) Ambra ${ }^{+/+}$and Ambra $1^{+/ g t}$ mice 7 days after ONC, $n=8$ animals per group. ${ }^{\#} p<0.01$ e $\%$ of RGCs stained with Brn3a in cryosections of old Ambra ${ }^{+/+}$and Ambra ${ }^{+/ g t}$ mice 7 days after ONC, $n=5$ animals per group. Data show mean $+/-\mathrm{SEM},{ }^{*} p<0.05$.

disease, including $\mathrm{ONC}^{49}$. Our data also shows that $\mathrm{Ambra1}^{+/+}$animals, but not the Ambra1 ${ }^{+/ g t}$ respond to these levels of oxidative stress by increasing the expression of the master antioxidant regulator Nrf2. These observations suggest a loop where increased ROS could exacerbate mitochondrial damage in the absence of an adequate antioxidant response after autophagy deficiency. Another pathway that protects cells against ROS is the elimination of whole damaged mitochondria via autophagy during mitophagy $^{50,51}$. Several lines of evidence suggest that AMBRA1 is a mitophagy receptor that can act in both PINK1/PARKIN dependent and independent pathways ${ }^{31,32}$. While some studies have found mitophagy events in RGCs after optic nerve transection $^{36}$ it is currently unknown how this pathway affects RGCs survival and which mitophagy regulators could be playing a role. Therefore, we decided to assess mRNA expression of several mitophagy regulators after ONC. Seven days after ONC we could detect an increase in Bnip3l and Bnip3 in the Ambra1 ${ }^{+/+}$animals, that was not observed in the $A m b r a 1^{+/ g t}$, Fig. 4. Conversely, Ambra1 ${ }^{+/ g t}$ did not show any changes of mitophagy regulators 3 or 7 days after ONC, Fig. 4c-f. Other mitophagy regulators such as Optn1, Park2, 


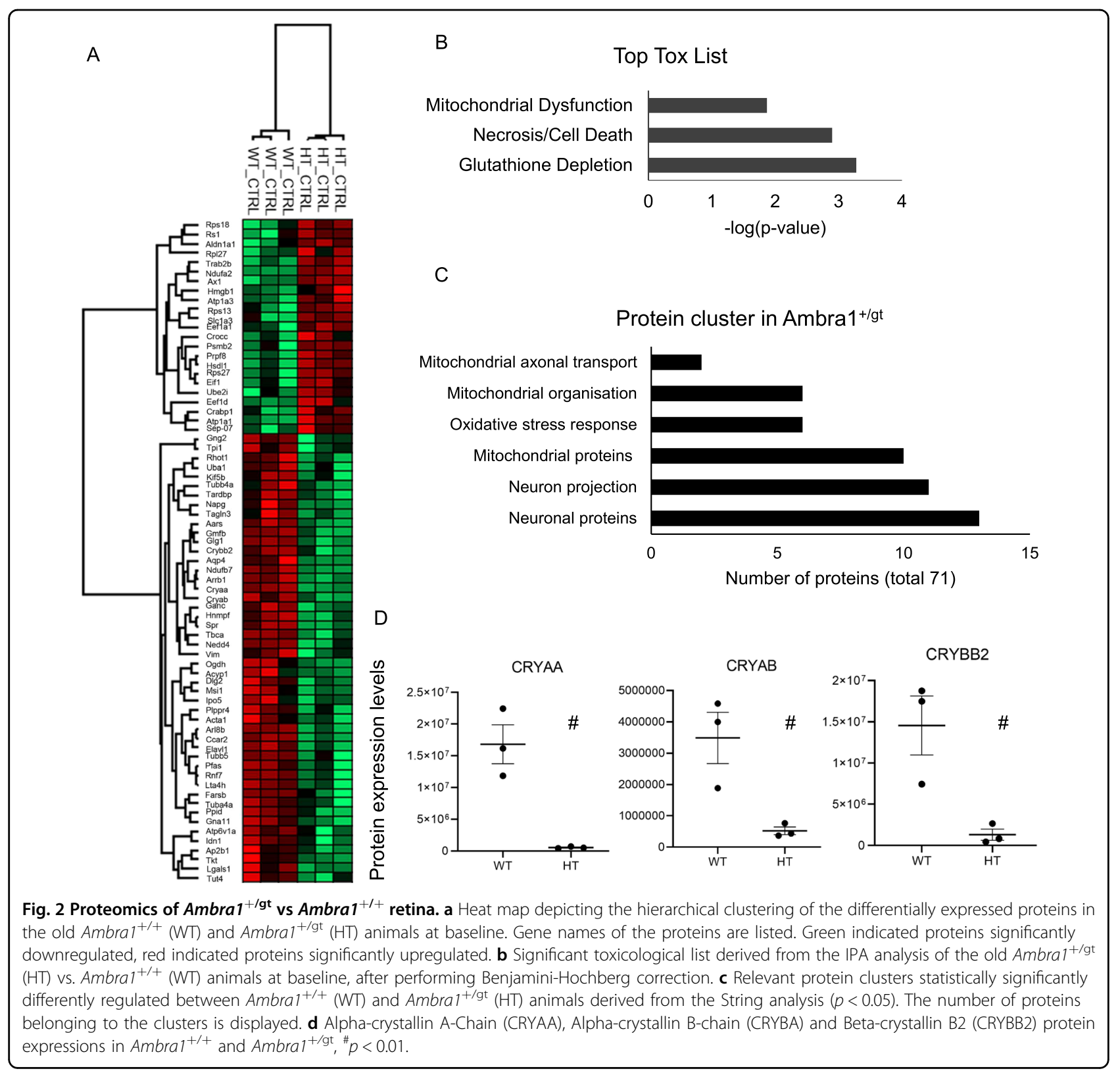

Pink1, and Mfn2 showed no changes. Interestingly, when we assessed the mRNA expression in the non-injured eye, we found that $A m b r a 1^{+/ g t}$ mice already displayed increased mRNA expression of both Bnip3l, Nrf2 and Mfn2 (Fig. 5), suggesting an increased protective response in the absence of axonal damage. This data could then suggest that the Ambra1 $1^{+/ g t}$ display an attempt to increase protective pathways which possibly cannot be further upregulated after an additional stress such as axonal damage. We therefore can show, that old $A m b r a 1^{+/ g t}$ mice display a misbalance in the oxidative stress regulation as well as mitochondrial proteins in the non-injured retina, which could be leading to a higher vulnerability status of the RGCs after injury.
Increased apoptosis and decreased neurite growth in Ambra1 ${ }^{+/ g t}$ RGCs

So far, the field of RGC research is still in need of a robust RGCs in vitro model to study alterations in the context of autophagy or mitophagy deficiency. We used a previously optimised protocol for RGC cultures from neonatal mice to study primary RGCs derived from $A m b r a 1^{+/ g t}$ and Ambra1 $1^{+/+}$mice in vitro. We isolated RGCs from retinas of neonatal $A m b r a 1^{+/ g t}$ and $A m b r a 1^{+/+}$mice and determined their morphology and survival. We observed an increase in the number of apoptotic cells in the $A m b r a 1^{+/ g t}$ derived cells in comparison to the Ambra1 $1^{+/+}$cells, Fig. 6a. Counting the number of RGCs in the culture, we found that 


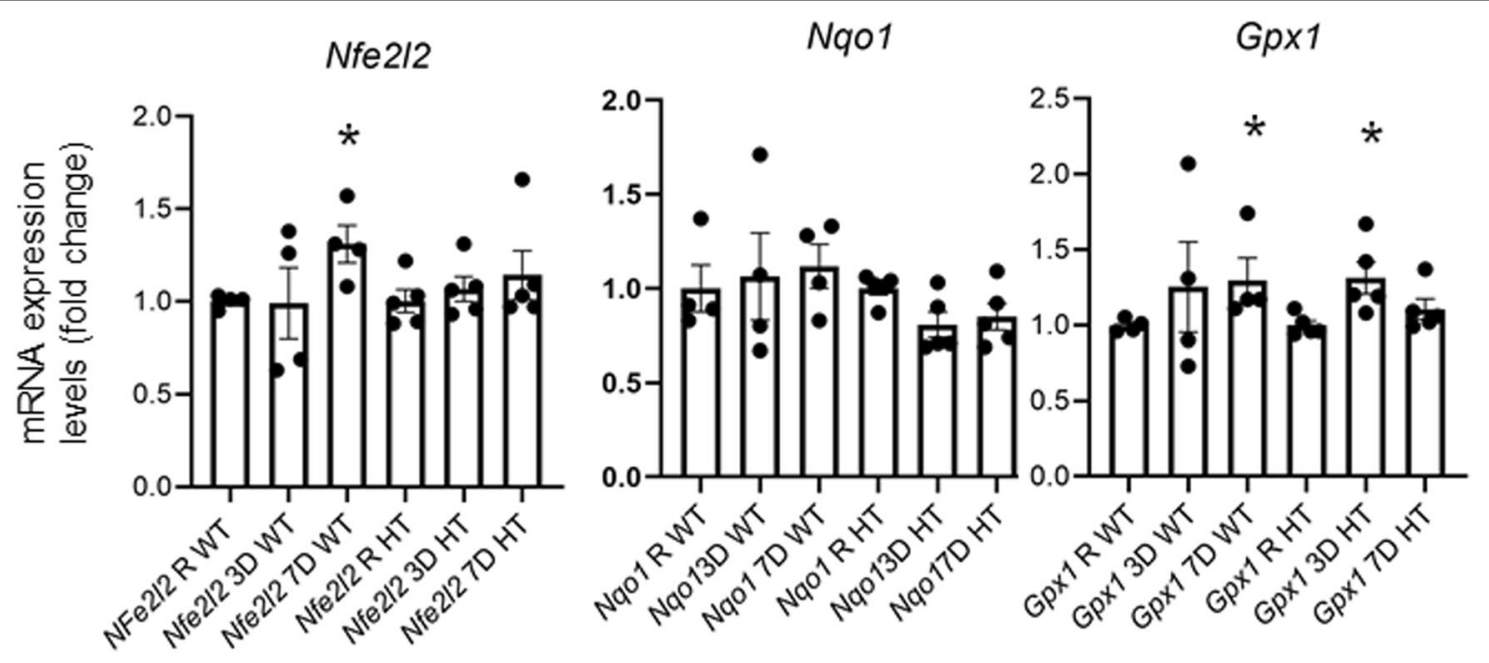

Fig. 3 Impaired oxidative stress response in old $\mathbf{A m b r a 1}^{+/ \mathbf{g t}}$ after ONC. mRNA expression levels determined by qPCR of Nfe2/2, Gpx1 and Nqo1 were measured in old $\mathrm{Ambral}^{+/+}$and Ambral ${ }^{+/ \mathrm{gt}}$ mice retinas 3 and 7 days after ONC. Data represent mean $+/-\mathrm{SEM},{ }^{*} \mathrm{p}<0.05$ in comparison to the corresponding control eye, $n=4-5$ per group.

Ambra1 $1^{+/ g t}$ derived cells had significantly less RGCs than Ambra1 ${ }^{+/+}$cultures, Fig. 6b, c. Additionally, the RGCs in the $\mathrm{Ambra1}^{+/ \mathrm{gt}}$ cultures displayed significantly more apoptotic nuclei, Fig. 6d. Last, the number of neuronal projections was comparable in both the $A m b r a 1^{+/ g t}$ and $\mathrm{Ambra1}^{+/+}$RGCs, however the length of outgrowing projections was significantly decreased in the Ambra1 ${ }^{+/ g t}$ derived RGCs, Fig. 6e. Our results demonstrate that primary RGCs derived from $\mathrm{Ambra1}^{+/ \mathrm{gt}}$ retinae show a higher vulnerability shown by increased rates of apoptosis. Furthermore, the ability of neurite outgrowth is significantly reduced in $\mathrm{Ambra1}^{+/ \mathrm{gt}}$ derived primary RGCs.

\section{Discussion}

In this study we have demonstrated an age dependent decrease in RGC survival after ONC in the Ambra1 ${ }^{+/ g t}$ mice compared to wildtype littermates. This is especially striking as we used middle aged animals (13 months of age), which do not display any apparent phenotype at this age. Mitochondrial alterations with impaired stress related mitophagy induction and altered oxidative stress response could play a role in the increased RGC susceptibility in the old $\mathrm{Ambra1}^{+/ \mathrm{gt}}$ animals. This is supported by our proteomic data as well as mRNA transcription for mitochondrial and oxidative stress genes. Our in vitro data additionally suggest a role for AMBRA1 in RGC survival and neurite outgrowth.

We demonstrate increased RGC loss in 13-month-old Ambra1 $1^{+/ g t}$ in comparison to Ambra1 ${ }^{+/+}$littermates and to the young experimental groups. We can rule out a general age-related decline in RGC survival, as the old Ambra1 $1^{+/+}$did not show any changes in RGC loss after $\mathrm{ONC}$ in comparison to the young Ambra1 $1^{+/+}$. Only few studies have analysed age related RGC susceptibility after ONC however, reduced RGC numbers after ONC has been shown for older animals, such as a study showing increased RGC damage after ONC for 24-month-old mice in comparison to young animals ${ }^{52}$. Regarding $A m b r a 1^{+/ g t}$ no studies have been performed analysing the agingrelated phenotype, but we did not observe differences in the number of RGCs in non-injured retinas (data not shown). Recent studies, however, have revealed that female $A \mathrm{mbra1}^{+/ g t}$ mice have some autistic features, but these are already apparent in young animals. Some of the analysed changes in the young animals however showed a slightly different characteristic in the middle aged mice, such as the form of induced seizures ${ }^{53,54}$, pointing towards a possible age related change. In our study we used a female to male ratio of 1:1 with an outbred CD1 background, whereas the studies showing autistic features were performed in $A m b r a 1^{+/ g t}$ with a C57BL/6N background and observations were only found in the female mice. Another study analysing neurogenesis in adult mice could demonstrate the importance of the Ambra1/Beclin 1 autophagy pathway in this context ${ }^{55}$. Increased RGC death after a severe ischaemia/reperfusion model, induced by increasing IOP and causing ischaemia of the retina for 60 mins, has been demonstrated previously in the Ambra1 $1^{+/ g t}$, the age of the animals was not stated in the paper $^{56}$. None of the studies showed an age-related impact of $A m b r a 1^{+/ g t}$. With our results we therefore are the first to demonstrate an age related increase in RGC vulnerability in the context of autophagy deficiency, leading to the conclusion that Ambra1 ${ }^{+/ g t}$ mice could be a perfect model to study age related neurodegenerative diseases in the context of autophagy deficiency. 


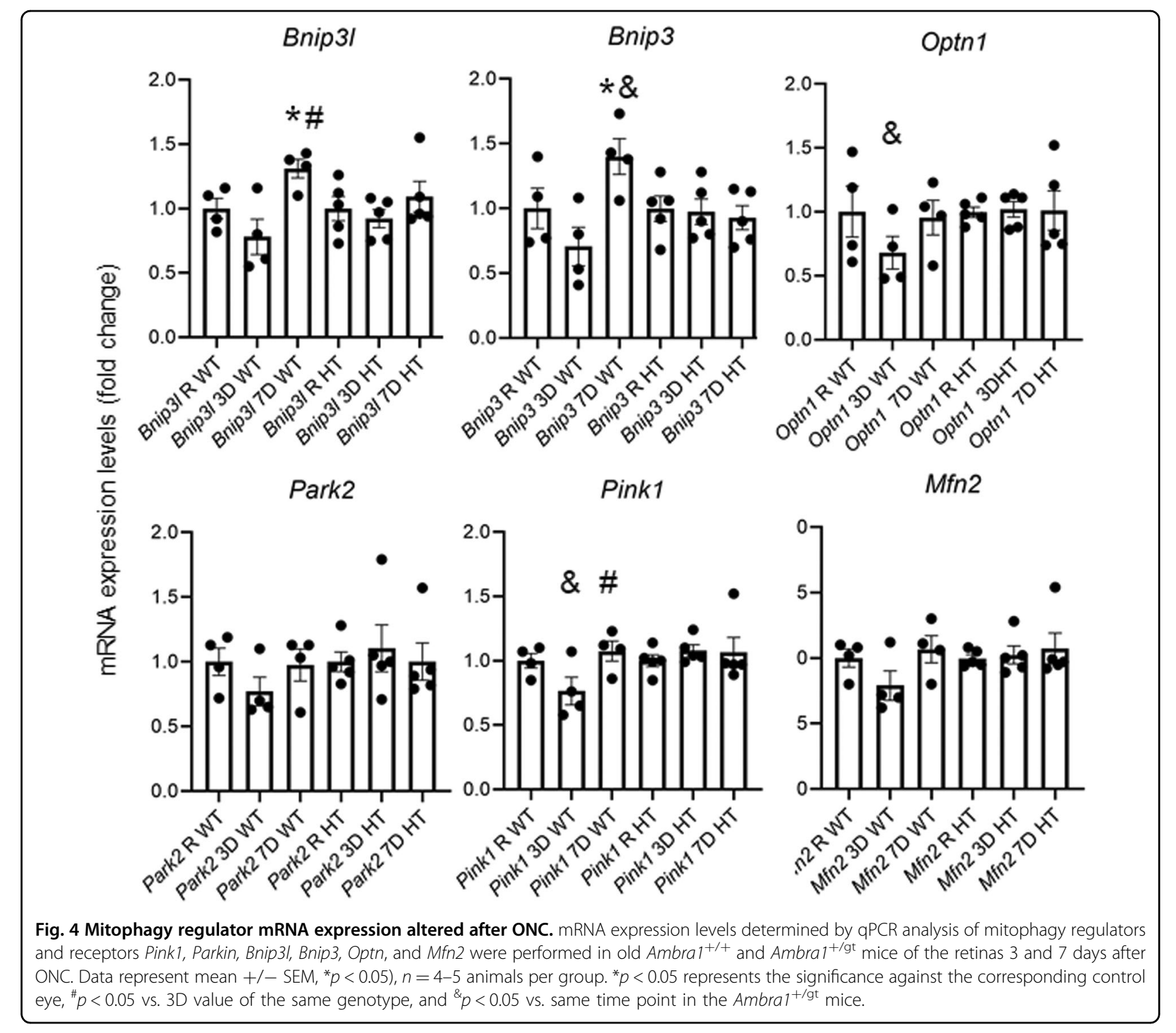

Our experiments show an altered oxidative stress response in $A m b r a 1^{+/ g t}$ animals, which could contribute to increased RGC loss after ONC in the aged Ambra $1^{+/ g t}$ animals. $8.5 \%$ of the proteins significantly differently regulated in the Ambra1 $1^{+/ g t}$ animals are involved in the oxidative stress response. Glutathione depletion was the most significantly altered toxicological function pathway found in the IPA analysis in the aged $A m b r a 1^{+/ g t}$ retinae, pointing towards dysregulation of this pathway in the retinae of these animals. Glutathione is one of the most prominent intracellular antioxidant systems ${ }^{57}$ that it is known to decline with age and promote stress-induced premature senescence in RPE cells ${ }^{58}$. Ambra1 $1^{+/ g t}$ animals show altered oxidative stress response after ONC displaying reduced mRNA Gpx1 induction in Ambra1 ${ }^{+/ g t}$ animals, and the failure to increase Nrf2 (Nfe2l2) mRNA or to adequately maintain Nqo1 mRNA levels sufficiently after ONC. GPX1 belongs to the very potent group of hydroperoxide-detoxifying enzymes, protecting the cell against oxidative damage such as offering a first line response for tackling mitochondrial derived $\operatorname{ROS}^{59,60}$. Briefly, upon overload of the GPX1 response, ROS accumulate and transcription of antioxidant genes such as the downstream NRF2 genes are activated. Increased Nrf2 expression promotes RGC survival after ONC and also plays a role in protecting RGCs against IOP related damage ${ }^{61,62}$. Additionally, Nrf2 knockout mice have also been shown to exacerbate optic neuritis in an experimental autoimmune encephalomyelitis model as well as in an ocular ischaemia and reperfusion model $^{63,64}$. NQO1 is another important antioxidant protein and can transcriptionally be regulated via Nrf2, as well as Aryl hydrocarbon receptor (AhR), although this does not necessarily lead to the same expression pattern ${ }^{65}$. Another study analysing Nqo1 mRNA expression found a slight increase 1 day after $\mathrm{ONC}$, however 4 and 7 days after ONC, the levels 


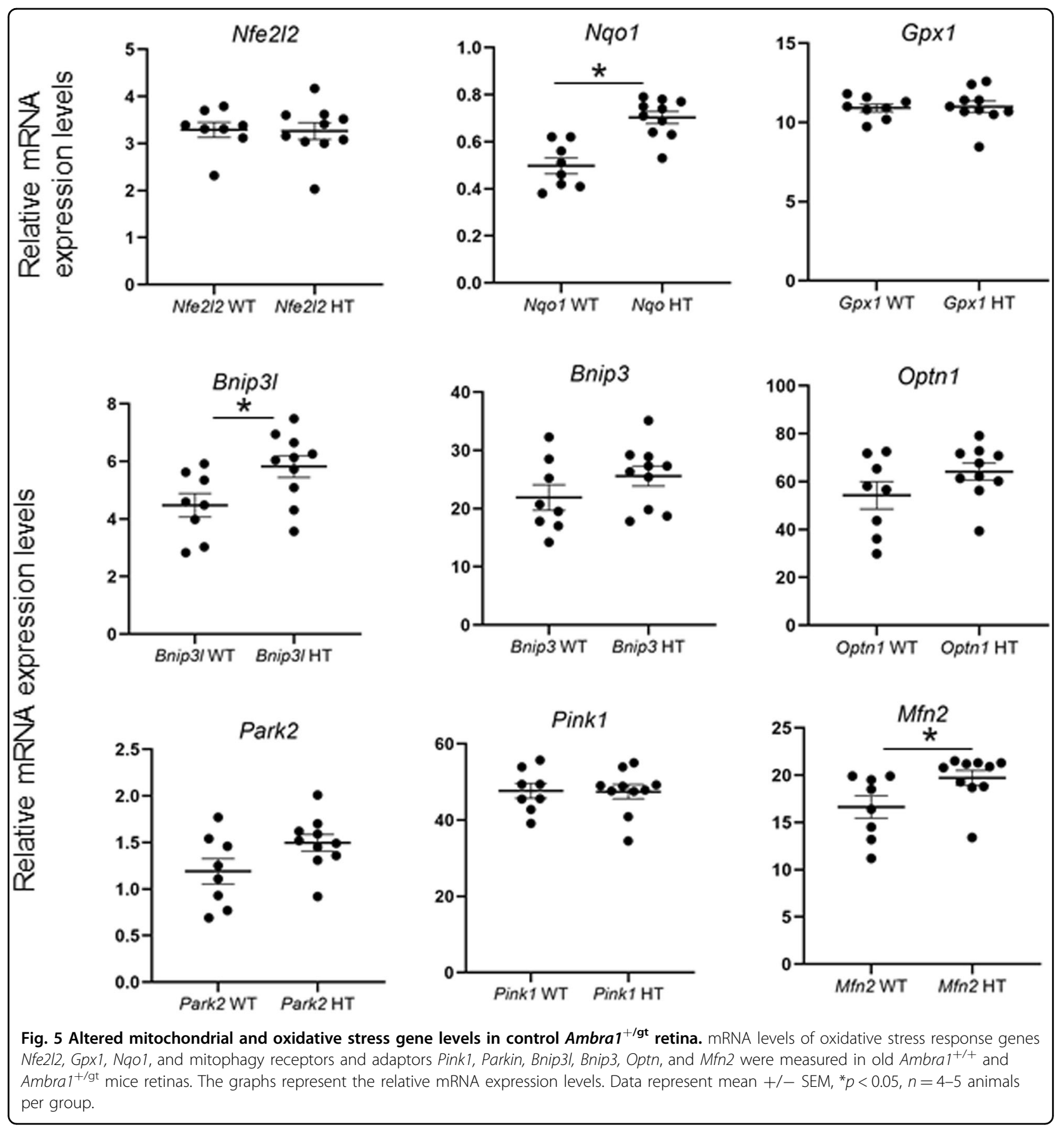

were back to baseline ${ }^{66}$. We believe that impaired oxidative stress response plays an important role for higher RGC vulnerability after damage in the aged $\mathrm{Ambra1}^{+/ g t}$ autophagy impaired animals. We furthermore conclude, that noninjured $\mathrm{Ambra1}^{+/ \mathrm{gt}}$ animals already have a higher activity of stress response mechanisms, due to autophagy deficiency. These chronically induced pathways could lead to an exhaustion of the system. If additional factors such as age in combination with more severe damage, as performed with the $\mathrm{ONC}$, are present, these pathways fail to protect the RGC.

Mitochondria are the main source of ROS within the cell and mitophagy is not only induced by oxidative stress but also plays a major role in modulating ROS in the cell ${ }^{67,68}$. More specifically, AMBRA1 mediated mitophagy plays an important role in counteracting oxidative stress in neuronal cells $^{69}$. Our results suggest increased mitophagy induction (Bnip3 and Bnip3l) in Ambra1 $1^{+/+}$animals) 7 days after 
A

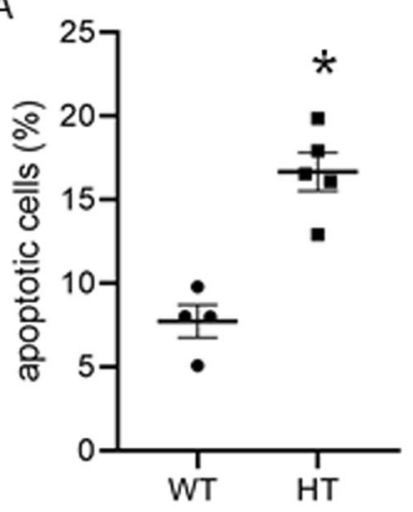

$\mathrm{C}$
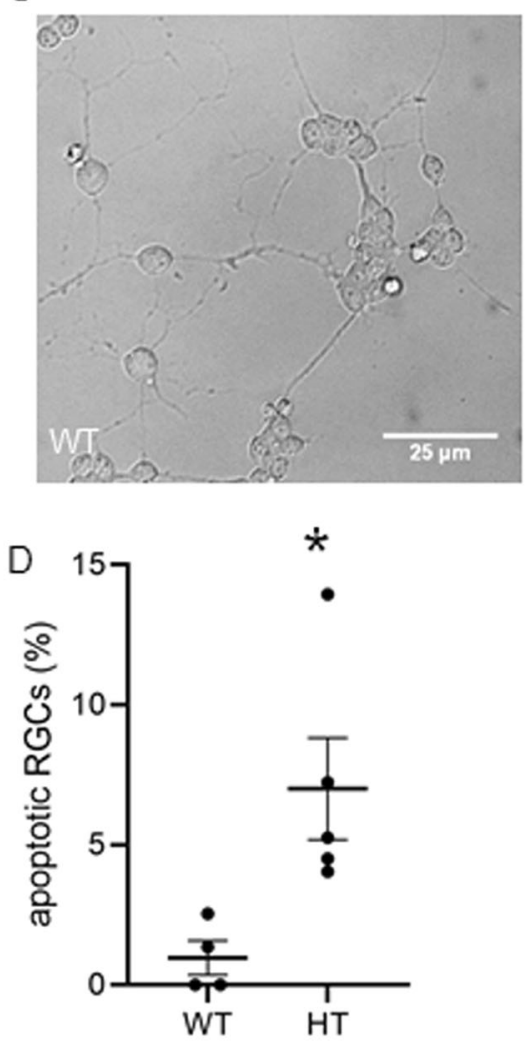

B
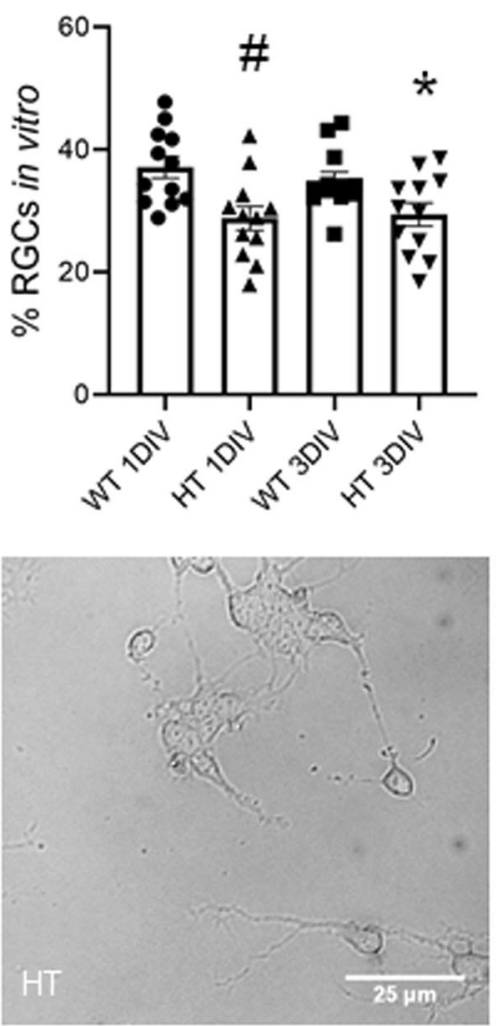

$E$

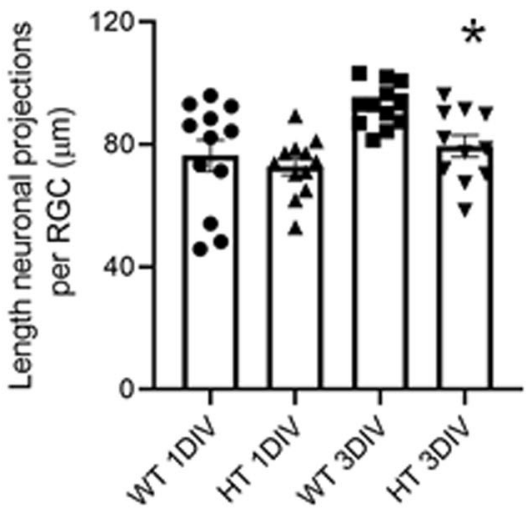

Fig. 6 Increased RGC apoptosis and decreased neuronal growth in Ambra $\mathbf{1}^{+/ \mathbf{g t}}$ derived RGCs. a \% of apoptotic cells determined by counting condensed nuclei in $\mathrm{Ambra1}^{+/+}$and Ambra1 ${ }^{+/ g t}$ retinal cell cultures ( $n=4$ for WT, $n=5$ for $H T$ ), ${ }^{*} p<0.01$, referring to comparison with WT, Data represent mean $+/$ - SEM. b RGC numbers in culture were determined 1 and 3 DIV using DIC pictures. The graph shows the number of RGCs, $n=15$. ${ }^{\#} p<0.01,{ }^{*} p<0.05$. Data represent mean +/- SEM. c Representative DIC pictures of the RGCs cultures after 3DIV. The scale bar $25 \mu \mathrm{m}$. $\mathbf{d} \%$ of apoptotic RGCs in culture in Ambra $^{+/+}$and Ambra $^{+/ 9 t}$ Apoptotic RGCs were counted using DAPI and TUJ1 staining $\left(n=4\right.$ for WT, $n=5$ for HT), ${ }^{*} p$ $<0.05$, in comparison to the corresponding WT value. e The length of RGC neurites was measured manually in DIC pictures in Ambra ${ }^{+/+}$and Ambra ${ }^{+/ g t}$. The graph represents the length of neuronal projection that each RGC grows at 1 DIV and 3 DIV, $n=15,{ }^{*} p<0.05$, referring to comparison with corresponding WT value, data represent mean +/- SEM.

ONC, whereas those levels are not increased in the Ambra1 ${ }^{+/ g t}$ animals. Increased Bnip3 levels 7 days have also been demonstrated in other CNS damage models such as in acute CNS ischaemia ${ }^{70}$ and induction of mitophagy via Bnip3l showed neuroprotective effects in acute cerebral damage independently of $\mathrm{PRKN}^{71,72}$. More generally, 
rapamycin has demonstrated beneficial effects in the context of acute CNS damage showing increased neuronal survival after spinal cord or brain injury as well as RGC rescue after optic nerve transection and ischaemia/reperfusion $^{36,56}$. Rapamycin is well known to increase autophagy, and also upregulates mitophagy and mitochondrial fission in in vivo CNS damage models ${ }^{73-76}$. Supporting possible increase of mitophagy after ONC, EM pictures of RGCs after optic nerve transection show increased numbers of mitochondria in autolysosomes 6 days after the insult ${ }^{36}$. It is tempting to speculate that increasing mitophagy therefore could possibly have a beneficial role for optic nerve damage and glaucoma.

We can show that primary RGCs derived from postnatal $A m b r a 1^{+/ g t}$ animals show less survival and reduced neurite outgrowth in vitro in comparison to Ambra1 ${ }^{+/+}$derived RGCs. Eleven of the proteins found to be differently regulated in $\mathrm{Ambral}^{+/ \mathrm{gt}}$ animals are involved in neuron projection and 7 proteins are involved in eye development. AMBRA1 plays an important role in embryonic neuronal development, leading to early embryonic death when totally knocked out ${ }^{30}$. However, Ambra1 ${ }^{+/ g t}$ animals show normal development and no apparent phenotype. Studies have demonstrated the role of AMBRA1 induced autophagy in the development of embryonic olfactory bulb neurons. Using eOBSCs (Stem/progenitor cells from the mouse embryo olfactory bulb) it was demonstrated that $A m b r a 1^{+/ g t}$ stem cells grow less and generate smaller neurospheres and less neurons, underlining the importance for AMBRA1 in neurons in a developmental stage ${ }^{34}$.

Our proteomics data showed decreased expression of several crystallin proteins (CRYAA, CRYAB, and CRYBB2) in Ambra1 ${ }^{+/ g t}$ retinae. Alpha-crystallins display chaperone like features, belong to the small heat shock family $^{77}$ and can protect cells from stress induced apoptosis $^{78}$. Alpha-crystallin expression in the retina is increased in various stress conditions such as diabetic retinopathy or glaucoma, aiming to protect the retinal cells from cell death ${ }^{4,79}$. All of the crystallins found in this study are predominantly expressed in $\mathrm{RGCs}^{44}$. More importantly, alpha-crystallins show RGC protective effects after $\mathrm{ONC}^{80}$ and neuroprotective effects of beta-crystallin B2 have been extensively studied in the context of RGC damage and survival models, as well as RGC axonal outgrowth $^{45,81}$. Additionally, crystallins can also be linked to age related macular degeneration, and altered crystallins lead to impaired lysosomal clearance in the retinal pigment epithelium ${ }^{82,83}$, however they have not been linked to AMBRA1 so far. Studies have been able to link crystallins (CRYBA1 and CRAYAB) to ATP6V1A expression. ATP6Va1 is a subunit of the vacuolar H+ATPase (VATPase) necessary for lysosomal acidification and also for autophagy ${ }^{84,85}$. Our proteomics analysis shows decreased ATP6Va1 in Ambra1 ${ }^{+/ g t}$ retinae, as well as other proteins involved in phagosome maturation. Thus, decreased crystallins in Ambra1 ${ }^{+/ g t}$ mice could not only lead to a loss of neuroprotective functions but also to defective lyosomal function and autophagy arrest. In addition, crystallins increase axonal growth in embryonic as well as young RGCs and neurons in vitro promoting neurite and axonal growth ${ }^{86,87}$ and reduced expression of these proteins could be playing a role in decreased RGC neurite outgrowth of $A m b r a 1^{+/ g t}$ derived RGCs.

In conclusion, we have shown an age-related increase in RGC susceptibility in the context of autophagy deficiency. Altered mitochondrial and oxidative stress response proteins in control but also after ONC conditions play a role in increased RGC vulnerability. It can be speculated that in control conditions, old Ambra1 ${ }^{+/ g t}$ mice have increased levels of defence pathways such as the oxidative stress response, resulting in an exhaustion of the system that leads to a reduced response after additional stress conditions. Future research should analyse in more detail the relevance of mitophagy and oxidative stress after neuronal damage to evaluate possible therapeutic targets for glaucoma and other related diseases.

\section{Acknowledgements \\ This work has been supported by grant PGC2018-098557-B-100 from Agencia Estatal de Investigacion from the Ministerio de Ciencia, Innovación e Universidades and EU. Katharina Bell receives support from DFG (Deutsche Forschungsgemeinschaft) Grant: 6619/1-1. \\ Author details \\ 'Department of Cellular and Molecular Biology, Centro de Investigaciones Biológicas Margarita Salas, CSIC, Madrid, Spain. ${ }^{2}$ Experimental and Translational Ophthalmology, Medical Center of the Johannes Gutenberg University, Mainz, Germany. ${ }^{3}$ Deutsche Zentrum für Neurodegenerative Erkrankungen e.V, DZNE/ German Center for Neurodegenerative Diseases, Dresden, Germany. \\ ${ }^{4}$ Department of Biology, University of Rome Tor Vergata, 00133 Rome, Italy}

Conflict of interest

The authors declare that they have no conflict of interest.

\section{Publisher's note}

Springer Nature remains neutral with regard to jurisdictional claims in published maps and institutional affiliations.

The online version of this article (https://doi.org/10.1038/s41420-020-0257-4) contains supplementary material, which is available to authorized users.

Received: 7 February 2020 Accepted: 25 March 2020

Published online: 17 April 2020

\section{References}

1. Tham, Y. C. et al. Global prevalence of glaucoma and projections of glaucoma burden through 2040: a systematic review and meta-analysis. Ophthalmology 121, 2081-2090 (2014).

2. Mirzaei, M. et al. Age-related neurodegenerative disease associated pathways identified in retinal and vitreous proteome from human glaucoma eyes. Sci. Rep. 7, 12685 (2017).

3. Van Bergen, N. J. et al. Measurement of systemic mitochondrial function in advanced primary open-angle glaucoma and leber hereditary optic neuropathy. PLOS ONE 10, e0140919 (2015). 
4. Funke, S. et al. Glaucoma related proteomic alterations in human retina samples. Sci. Rep. 6, 29759 (2016).

5. Boya, P., Reggiori, F. \& Codogno, P. Emerging regulation and functions of autophagy. Nat. Cell Biol. 15, 713-720 (2013).

6. Klionsky, D. J., Eskelinen, E. L. \& Deretic, V. Autophagosomes, phagosomes, autolysosomes, phagolysosomes, autophagolysosomes... wait, I'm confused. Autophagy 10, 549-551 (2014).

7. Kirkin, V. \& Rogov, V. V. A diversity of selective autophagy receptors determines the specificity of the autophagy pathway. Mol. Cell 76, 268-285 (2019).

8. Pattison, C. J. \& Korolchuk, V. I. Autophagy: 'Self-Eating' your way to longevity. Sub-Cell. Biochem. 90, 25-47 (2018).

9. Revuelta, M. \& Matheu, A. Autophagy in stem cell aging. Aging Cell 16, 912-915 (2017).

10. Lipinski, M. M. et al. Genome-wide analysis reveals mechanisms modulating autophagy in normal brain aging and in Alzheimer's disease. Proc. Natl Acad. Sci. USA 107, 14164-14169 (2010).

11. Rodriguez-Muela, N. et al. Balance between autophagic pathways preserves retinal homeostasis. Aging Cell 12, 478-488 (2013).

12. Hara, T. et al. Suppression of basal autophagy in neural cells causes neurodegenerative disease in mice. Nature 441, 885-889 (2006).

13. Komatsu, M. et al. Loss of autophagy in the central nervous system causes neurodegeneration in mice. Nature 441, 880-884 (2006).

14. Metaxakis, A., Ploumi, C. \& Tavernarakis, N. Autophagy in age-associated neurodegeneration. Cells. https://doi.org/10.3390/cells7050037 (2018).

15. Kuma, A. et al. The role of autophagy during the early neonatal starvation period. Nature 432, 1032-1036 (2004).

16. Yoshii, S. R. et al. Systemic analysis of Atg5-null mice rescued from neonatal lethality by transgenic ATG5 expression in neurons. Dev. Cell 39, 116-130 (2016).

17. Mellen, M. A., de la Rosa, E. J. \& Boya, P. The autophagic machinery is necessary for removal of cell corpses from the developing retinal neuroepithelium. Cell Death Differ. 15, 1279-1290 (2008).

18. Esteban-Martinez, L. \& Boya, P. BNIP3L/NIX-dependent mitophagy regulates cell differentiation via metabolic reprogramming. Autophagy 14, 915-917 (2018).

19. Wiggs, J. L. Glaucoma genes and mechanisms. Prog. Mol. Biol. Transl. Sci. 134, 315-342 (2015).

20. Swarup, G. \& Sayyad, Z. Altered functions and interactions of glaucomaassociated mutants of optineurin. Front. Immunol. 9, 1287 (2018).

21. Sears, N. C., Boese, E. A., Miller, M. A. \& Fingert, J. H. Mendelian genes in primary open angle glaucoma. Exp. Eye Res. 186, 107702 (2019).

22. Aung, $T$. et al. Investigating the association between OPA1 polymorphisms and glaucoma: comparison between normal tension and high tension primary open angle glaucoma. Hum. Genet. 110, 513-514 (2002).

23. Aung, T. et al. A major marker for normal tension glaucoma: association with polymorphisms in the OPA1 gene. Hum. Genet. 110, 52-56 (2002).

24. Hu, X., Dai, Y., Zhang, R., Shang, K. \& Sun, X. Overexpression of optic atrophy type 1 protects retinal ganglion cells and upregulates Parkin expression in experimental glaucoma. Front. Mol. Neurosci. 11, 350 (2018).

25. Choquet, H., Wiggs, J. L. \& Khawaja, A. P. Clinical implications of recent advances in primary open-angle glaucoma genetics. Eye 34, 29-39 (2020).

26. Youngblood, H., Hauser, M. A. \& Liu, Y. Update on the genetics of primary open-angle glaucoma. Exp. Eye Res. 188, 107795 (2019).

27. Lazarou, M. et al. The ubiquitin kinase PINK1 recruits autophagy receptors to induce mitophagy. Nature 524, 309-314 (2015).

28. Lang, A. et al. SIRT4 interacts with OPA1 and regulates mitochondrial quality control and mitophagy. Aging 9, 2163-2189 (2017).

29. Song, B. J. \& Caprioli, J. New directions in the treatment of normal tension glaucoma. Indian J. Ophthalmol. 62, 529-537 (2014).

30. Fimia, G. M. et al. Ambra1 regulates autophagy and development of the nervous system. Nature 447, 1121-1125 (2007).

31. Van Humbeeck, C. et al. Parkin interacts with Ambra1 to induce mitophagy. J. Neurosci. 31, 10249-10261 (2011)

32. Strappazzon, F. et al. AMBRA1 is able to induce mitophagy via LC3 binding regardless of PARKIN and p62/SQSTM1. Cell Death Differ. 22, 419-432 (2015).

33. Di Rita, A. et al. HUWE1 E3 ligase promotes PINK1/PARKIN-independent mitophagy by regulating AMBRA1 activation via IKKalpha. Nat. Commun. 9, 3755 (2018).

34. Vazquez, P. et al. Atg5 and Ambra1 differentially modulate neurogenesis in neural stem cells. Autophagy 8, 187-199 (2012).
35. Rodriguez-Muela, $\mathrm{N}$. et al. Lysosomal membrane permeabilization and autophagy blockade contribute to photoreceptor cell death in a mouse model of retinitis pigmentosa. Cell Death Differ. 22, 476-487 (2015).

36. Rodriquez-Muela, N., Germain, F., Marino, G., Fitze, P. S. \& Boya, P. Autophagy promotes survival of retinal ganglion cells after optic nerve axotomy in mice. Cell Death Differ. 19, 162-169 (2012).

37. Schmelter, C. et al. Comparison of two solid-phase extraction (SPE) methods for the identification and quantification of porcine retinal protein markers by LC-MS/MS. Int. J. Mol. Sci. https://doi.org/10.3390/ijms19123847 (2018).

38. Schmelter, C. et al. Synthetic polyclonal-derived CDR peptides as an innovative strategy in glaucoma therapy. J. Clin. Med. https://doi.org/10.3390/jcm8081222 (2019).

39. Schmelter, C. et al. Peptides of the variable lgG domain as potential biomarker candidates in primary open-angle glaucoma (POAG). Hum. Mol. Genet. 26 4451-4464 (2017).

40. Funke, S. et al. Comparative quantitative analysis of Porcine optic nerve head and retina subproteomes. Int. J. Mol. Sci. https://doi.org/10.3390/ijms20174229 (2019).

41. Tyanova, S. et al. The Perseus computational platform for comprehensive analysis of (prote)omics data. Nat. Methods 13, 731-740 (2016).

42. Surgucheva, I., Weisman, A. D., Goldberg, J. L., Shnyra, A. \& Surguchov, A. Gamma-synuclein as a marker of retinal ganglion cells. Mol. Vis. 14, 1540-1548 (2008).

43. Nadal-Nicolas, F. M., Sobrado-Calvo, P., Jimenez-Lopez, M., Vidal-Sanz, M. \& Agudo-Barriuso, M. Long-term effect of optic nerve axotomy on the retinal ganglion cell layer. Investig. Ophthalmol. Vis. Sci. 56, 6095-6112 (2015).

44. Piri, N., Kwong, J. M. \& Caprioli, J. Crystallins in retinal ganglion cell survival and regeneration. Mol. Neurobiol. 48, 819-828 (2013).

45. Bohm, M. R. et al. betaB2-crystallin promotes axonal regeneration in the injured optic nerve in adult rats. Cell Transplant. 24, 1829-1844 (2015).

46. Sivandzade, F., Bhalerao, A. \& Cucullo, L. Cerebrovascular and neurological disorders: protective role of NRF2. Int. J. Mol. Sci. https://doi.org/10.3390/ ijms20143433 (2019).

47. Tonelli, C., Chio, I. I. C. \& Tuveson, D. A. Transcriptional regulation by Nrf2. Antioxid. redox Signal. 29, 1727-1745 (2018).

48. Pinazo-Duran, M. D. et al. Strategies to reduce oxidative stress in glaucoma patients. Curr. Neuropharmacol. 16, 903-918 (2018).

49. Li, H. Y., Ruan, Y. W., Ren, C. R., Cui, Q. \& So, K. F. Mechanisms of secondary degeneration after partial optic nerve transection. Neural Regen. Res. 9, 565-574 (2014).

50. Ashrafi, G. \& Schwarz, T. L. The pathways of mitophagy for quality control and clearance of mitochondria. Cell Death Differ. 20, 31-42 (2013).

51. Di Rita, A. \& Strappazzon, F. Mitophagy could fight Parkinson's disease through antioxidant action. Rev. Neurosci. https://doi.org/10.1515/revneuro-2018-0095 (2019).

52. Wang, A. L., Yuan, M. \& Neufeld, A. H. Age-related changes in neuronal susceptibility to damage: comparison of the retinal ganglion cells of young and old mice before and after optic nerve crush. Ann. N. Y. Acad. Sci. 1097, 64-66 (2007).

53. Dere, E. et al. Heterozygous ambra1 deficiency in mice: a genetic trait with autism-like behavior restricted to the female gender. Front. Behav. Neurosci. 8, 181 (2014).

54. Mitjans, M. et al. Sexual dimorphism of AMBRA1-related autistic features in human and mouse. Transl. Psychiatry 7, e1247 (2017).

55. Yazdankhah, M., Farioli-Vecchioli, S., Tonchev, A. B., Stoykova, A. \& Cecconi, F. The autophagy regulators Ambra1 and Beclin 1 are required for adult neurogenesis in the brain subventricular zone. Cell Death Dis. 5, e1403 (2014).

56. Russo, R. et al. Rapamycin and fasting sustain autophagy response activated by ischemia/reperfusion injury and promote retinal ganglion cell survival. Cell Death Dis. 9, 981 (2018).

57. De Nicola, M. \& Ghibelli, L. Glutathione depletion in survival and apoptotic pathways. Front. Pharmacol. 5, 267 (2014).

58. Sun, Y., Zheng, Y., Wang, C. \& Liu, Y. Glutathione depletion induces ferroptosis, autophagy, and premature cell senescence in retinal pigment epithelial cells. Cell Death Dis. 9, 753 (2018).

59. Lubos, E., Loscalzo, J. \& Handy, D. E. Glutathione peroxidase-1 in health and disease: from molecular mechanisms to therapeutic opportunities. Antioxid. Redox Signal. 15, 1957-1997 (2011).

60. De Haan, J. B. et al. An imbalance in antioxidant defense affects cellular function: the pathophysiological consequences of a reduction in antioxidant 
defense in the glutathione peroxidase-1 (Gpx1) knockout mouse. Redox Rep. 8 , 69-79 (2003).

61. Xiong, W., MacColl Garfinkel, A. E., Li, Y., Benowitz, L. I. \& Cepko, C. L. NRF2 promotes neuronal survival in neurodegeneration and acute nerve damage. J. Clin. Investig. 125, 1433-1445 (2015).

62. Wang, X. \& Yuan, Z. L. Activation of Nrf2/HO-1 pathway protects retinal ganglion cells from a rat chronic ocular hypertension model of glaucoma. Int. Ophthalmol. 39, 2303-2312 (2019).

63. Larabee, C. M. et al. Loss of Nrf2 exacerbates the visual deficits and optic neuritis elicited by experimental autoimmune encephalomyelitis. Mol. Vis. 22, 1503-1513 (2016)

64. $\mathrm{Xu}, \mathrm{Z}$. et al. Neuroprotective role of Nrf2 for retinal ganglion cells in ischemiareperfusion. J. Neurochem. 133, 233-241 (2015).

65. Ross, D. \& Siegel, D. Functions of NQO1 in cellular protection and CoQ10 metabolism and its potential role as a redox sensitive molecular switch. Front. Physiol. 8, 595 (2017).

66. Himori, N. et al. Critical role of Nrf2 in oxidative stress-induced retinal ganglion cell death. J. Neurochem. 127, 669-680 (2013).

67. Dai, D. F., Chiao, Y. A., Marcinek, D. J., Szeto, H. H. \& Rabinovitch, P. S. Mitochondrial oxidative stress in aging and healthspan. Longev. Healthspan 3, 6 (2014).

68. Frank, M. et al. Mitophagy is triggered by mild oxidative stress in a mitochondrial fission dependent manner. Biochim. et. Biophys. Acta 1823, 2297-2310 (2012)

69. Di Rita, A. et al. AMBRA1-mediated mitophagy counteracts oxidative stress and apoptosis induced by neurotoxicity in human neuroblastoma SH-SY5Y. Cells Front. Cell. Neurosci. 12, 92 (2018).

70. Ulamek-Koziol, M. et al. Dysregulation of autophagy, mitophagy, and apoptotic genes in the medial temporal lobe cortex in an ischemic model of Alzheimer's disease. J. Alzheimer's Dis. 54, 113-121 (2016).

71. Yuan, Y. et al. BNIP3L/NIX-mediated mitophagy protects against ischemic brain injury independent of PARK2. Autophagy 13, 1754-1766 (2017).

72. Ma, J. et al. Potential roles of NIX/BNIP3L pathway in rat traumatic brain injury. Cell Transplant. 28, 585-595 (2019).

73. Li, Q. et al. Rapamycin enhances mitophagy and attenuates apoptosis after spinal ischemia-reperfusion. Inj. Front. Neurosci. 12, 865 (2018).
74. Cavallucci, V. et al. Acute focal brain damage alters mitochondrial dynamics and autophagy in axotomized neurons. Cell death Dis. 5, e1545 (2014).

75. Wang, C. et al. The post-therapeutic effect of rapamycin in mild traumatic brain-injured rats ensuing in the upregulation of autophagy and mitophagy. Cell Biol. Int. 41, 1039-1047 (2017).

76. Li, Q. et al. Rapamycin attenuates mitochondrial dysfunction via activation of mitophagy in experimental ischemic stroke. Biochem. Biophys. Res. Commun. 444, 182-188 (2014).

77. Horwitz, J. Alpha-crystallin can function as a molecular chaperone. Proc. Nat/ Acad. Sci. USA 89, 10449-10453 (1992).

78. Raju, M., Santhoshkumar, P. \& Krishna Sharma, K. Alpha-crystallin-derived peptides as therapeutic chaperones. Biochim. et. Biophys. Acta 1860, 246-251 (2016).

79. Kase, S., Ishida, S. \& Rao, N. A. Increased expression of alphaA-crystallin in human diabetic eye. Int. J. Mol. Med. 28, 505-511 (2011).

80. Ying, $X$. et al. Alpha-crystallin protected axons from optic nerve degeneration after crushing in rats. J. Mol. Neurosci. 35, 253-258 (2008).

81. Anders, F. et al. Intravitreal injection of beta-crystallin B2 improves retinal ganglion cell survival in an experimental animal model of glaucoma. PLOS ONE 12, e0175451 (2017).

82. Sinha, D. et al. Lysosomes: regulators of autophagy in the retinal pigmented epithelium. Exp. eye Res. 144, 46-53 (2016).

83. Zigler, J. S. Jr. \& Sinha, D. betaA3/A1-crystallin: more than a lens protein. Prog. Retinal Eye Res. 44, 62-85 (2015).

84. Valapala, M. et al. Lysosomal-mediated waste clearance in retinal pigment epithelial cells is regulated by CRYBA1/betaA3/A1-crystallin via V-ATPaseMTORC1 signaling. Autophagy 10, 480-496 (2014).

85. Cui, X. et al. Heat shock factor 4 regulates lens epithelial cell homeostasis by working with lysosome and anti-apoptosis pathways. Int. J. Biochem. Cell Biol. 79, 118-127 (2016).

86. Lorber, B., Berry, M., Logan, A. \& Tonge, D. Effect of lens lesion on neurite outgrowth of retinal ganglion cells in vitro. Mol. Cell. Neurosci. 21, 301-311 (2002).

87. Liedtke, T., Schwamborn, J. C., Schroer, U. \& Thanos, S. Elongation of axons during regeneration involves retinal crystallin beta b2 (crybb2). Mol. Cell. Proteom. 6, 895-907 (2007) 